\title{
Some Explicit Krein Representations of Certain Subordinators, Including the Gamma Process
}

\author{
By \\ Catherine Donati-Martin* and Marc Yor**
}

\begin{abstract}
We give a representation of the Gamma subordinator as a Krein functional of Brownian motion, using the known representations for stable subordinators and Esscher transforms. In particular, we have obtained Krein representations of the subordinators which govern the two parameter Poisson-Dirichlet family of distributions [23].
\end{abstract}

\section{$\S 1$. Introduction}

(1.a) The aim of this paper is to represent explicitly a particular class of subordinators $\left(S_{t} ; t \geq 0\right)$, i.e. increasing $\mathbb{R}_{+}$-valued Lévy processes, as inverse local times of $\mathbb{R}_{+}$-valued diffusions. This problem was raised in Itô-Mc Kean [8], and completely solved, in a theoretical manner, thanks to Krein's representation of strings, by Knight [10] and Kotani-Watanabe [11] independently and simultaneously in 1981-1982.

Roughly, if the Lévy measure $\nu$ of $\left(S_{t} ; t \geq 0\right)$ admits a density $h$ with respect to the Lebesgue measure: $\nu(d y)=h(y) d y$ and if $h(y)=$ $\int_{0}^{\infty} \mu(d x) \exp (-y x)$ for some positive measure $\mu$, then the above mentioned problem, which we shall call the Krein representation problem, is solved in the affirmative. See, besides [10] and [11], a number of other papers dealing with this question, e.g. Bertoin ([1], [2]), Küchler [12], Küchler and Salminen [13].

Communicated by Y. Takahashi. Received April 27, 2005.

2000 Mathematics Subject Classification(s): 60G51, $60 J 55$.

*Laboratoire de Probabilités et Modèles Aléatoires, Université Paris 6, Site Chevaleret, 13 rue Clisson, F-75013 Paris.

e-mail: donati@ccr.jussieu.fr

** Laboratoire de Probabilités et Modèles Aléatoires, Université Paris 6, Site Chevaleret, 13 rue Clisson, F-75013 Paris. 
(1.b) In this paper, rather than discussing this problem in general, we solve it for the two parameter family of subordinators $\left(S_{t}^{\alpha, \beta} ; t \geq 0\right)$ whose Lévy measures are

$$
\nu_{\alpha, \beta}(d y)=C \frac{\exp (-\beta y)}{y^{\alpha+1}} d y, 0 \leq \alpha<1, \beta \geq 0
$$

(for $\alpha=0, \beta$ is assumed $>0$ ).

The constant $C$ plays a simple role and might, a priori, be suppressed from our discussion; however, it is in fact very helpful to keep this further degree of freedom in order to consider the most convenient local time at 0 (for the underlying diffusion), which, as is well known, may be chosen up to a multiplicative constant.

(1.c) It goes back at least to Molchanov-Ostrovski [20] that for $0<\alpha<1$, $S_{t}^{\alpha, 0}$, the stable subordinator of index $\alpha$, may be realized as the inverse local time of a Bessel process (which we shall denote as $B E S(-\alpha)$ ) of dimension $\delta=2(1-\alpha)$. Since, by inspection of the Lévy measures: $\nu_{\alpha, \beta}(d y)=$ $\exp (-\beta y) \nu_{\alpha, 0}(d y), S_{t}^{\alpha, \beta}$ may be obtained as an Esscher transform of $S_{t}^{\alpha, 0}$, it is natural to look for a Girsanov transform of $B E S(-\alpha)$ whose inverse local time is distributed as $S_{t}^{\alpha, \beta}$. As we show below, this is indeed the case with $B E S(-\alpha, \beta \downarrow)$ the downwards $B E S(-\alpha)$ process with "drift" $\beta$, following the terminology of Watanabe [31], [32] and Pitman-Yor [21].

Finally, the case $\alpha=0, \beta>0$, which corresponds to the Gamma process $\left(S_{t}^{0, \beta} ; t \geq 0\right)$ is obtained from the case $\alpha>0$ by letting $\alpha \longrightarrow 0$ in a suitable manner.

(1.d) It is also natural to look for some representation of these subordinators as continuous additive functionals of a Brownian motion $\left(B_{s} ; s \geq 0\right)$ taken at the inverse local time $\left(\tau_{t}^{*} ; t \geq 0\right)$ of that Brownian motion. This is done expressing the Bessel processes with drift in terms of their Feller representations, using scale functions and speed measures. For example, it was remarked in Biane-Yor [3] that:

$$
\left(\int_{0}^{\tau_{t}^{*}}\left|B_{u}\right|^{\frac{1}{\alpha}-2} d u, t \geq 0\right) \stackrel{(\text { law })}{=}\left(S_{t}^{\alpha, 0} ; t \geq 0\right),
$$

and that all symmetric stable Lévy processes may be obtained in this manner, replacing the even power $|x|^{\frac{1}{\alpha}-2}$ by the "symmetric" power i.e.: $\sigma(x)=$ $\operatorname{sgn}(x)|x|^{\frac{1}{\alpha}-2}$. For example, $\left(\frac{1}{\pi} \int_{0}^{\tau_{t}^{*}} \frac{d s}{B_{s}} ; t \geq 0\right)$ is a standard Cauchy process. 
More generally, every asymmetric stable Lévy process may be represented in a similar manner from Brownian motion. Although in the sequel, we shall also extend (1.1) to present in general $\left(S_{t}^{\alpha, \beta} ; t \geq 0\right)$ in terms of Brownian additive functionals taken at $\left(\tau_{t}^{*} ; t \geq 0\right)$, these presentations are not so simple, and we prefer to those the Krein representations evoked in (1.c).

(1.e) The rest of the paper is organized as follows. Our main Krein representation results are presented in Section 2. The full proofs are given in Section 3. The Brownian additive functional representations are discussed in Section 4. Finally, in Section 5, we also give some Krein representations of the symmetric Lévy processes on $\mathbb{R}$ (without Gaussian component) whose Lévy measures are given by:

$$
\tilde{\nu}_{\alpha, \beta}(d y)=\frac{\exp (-\beta|y|)}{|y|^{\alpha+1}} d y
$$

$(0 \leq \alpha<2, \beta>0)$

(1.f) As an end to this introduction, let us point out that, the subordinators $S^{\alpha, \beta}$ we represent here, and their symmetric counterparts, are arguably the most studied and used among Lévy processes, and this, for the following reasons: for $\alpha>0, S^{\alpha, \beta}$ is obtained by Esscher transform (see [7, 26]) from the fundamental stable $(\alpha)$ subordinator, hence it "retains" some scaling property, while the Gamma process ([6], [27], [28], [29], [30]) and the variance-gamma processes $([15],[16],[17])$ have some fundamental quasi-invariance properties, which make them comparable, in some respect, to Brownian motion with drift.

(1.g) Finally, we refer the reader to a systematic compendium [5] of constants $C_{\alpha}$ related to various choices found in the literature of local times with respect to $B E S(-\alpha)$. We also intend in [4] to study a number of properties of the symmetrized $B E S(-\alpha, \beta \downarrow)$ processes with a view towards a discussion of Krein representations for the variance-gamma processes.

\section{$\S 2 . \quad$ Definition of the $B E S(-\alpha, \beta \downarrow)$ Processes and Main Krein Representation Results}

We first recall that for $0<\alpha<1, B E S(-\alpha)$, which we call the Bessel process of index $-\alpha$, (or dimension $d=2(1-\alpha)$ ) is the $\mathbb{R}_{+}$-valued diffusion with infinitesimal generator

$$
\frac{1}{2} \frac{d^{2}}{d x^{2}}+\frac{1-2 \alpha}{2 x} \frac{d}{d x}
$$


which is instantaneously reflecting at 0 . We denote by $P_{x}^{(-\alpha)}$ its distribution on $C\left(\mathbb{R}_{+}, \mathbb{R}_{+}\right)$, where $R_{t}(\omega)=\omega(t), \mathcal{R}_{t}=\sigma\left\{R_{s} ; s \leq t\right\}$ and $R_{0}=x$.

For the sequel, it will be convenient to introduce the parameter $\theta=\sqrt{2 \beta}$ for $\beta>0$. We may now define $B E S(-\alpha, \beta \downarrow)$ as the diffusion with law $\left(P_{x}^{(-\alpha), \beta \downarrow}\right)$, obtained by Girsanov ${ }^{1}$ transform from $B E S(-\alpha)$ :

$$
\left.P_{x}^{(-\alpha), \beta \downarrow}\right|_{\mathcal{R}_{t}}=\left.\frac{\hat{K}_{\alpha}\left(\theta R_{t}\right)}{\hat{K}_{\alpha}(\theta x)} \exp \left(C_{\alpha}(2 \beta)^{\alpha} l_{t}-\beta t\right) P_{x}^{(-\alpha)}\right|_{\mathcal{R}_{t}}
$$

where $\hat{K}_{\alpha}(x)=x^{\alpha} K_{\alpha}(x), x \geq 0$, which satisfies $\hat{K}_{\alpha}(0)=2^{\alpha-1} \Gamma(\alpha)$ and $\left(l_{t} ; t \geq\right.$ $0)$ is a choice of the local time at level 0 of $R$ made so that the following holds: Itô's excursion measure $n_{\alpha}^{\beta \downarrow}(d e)$ associated with $B E S(-\alpha, \beta \downarrow)$ together with this choice of local time may be described as follows:

a) $n_{\alpha}^{\beta \downarrow}(V(e) \in d v)=\frac{2^{\alpha} \Gamma(\alpha+1)}{v^{\alpha+1}} \exp (-\beta v) d v$, where $V(e)$ denotes the lifetime of the generic excursion $e$.

b) Conditionally on $V=v$, the process $(e(u), u \leq v)$ is distributed as a Bessel bridge of index $\alpha$, and length $v$.

Note that this description is valid, in particular, for $\beta=0$.

We also refer the reader to paragraph 5.4 in which, in parallel with these precisions for $n_{\alpha}^{\beta \downarrow}$, we have specified density of occupation formulae for the $B E S(-\alpha, \beta \downarrow)$ processes. The conditioned diffusions $B E S(-\alpha, \beta \downarrow)$, started at $x>0$ and killed at $T_{0}$ are described in [21]. See also Watanabe [31, 32, 33].

We now discuss some immediate consequences of these choices: from (a), it follows that:

$$
E_{0}^{(-\alpha), \beta \downarrow}\left(\exp \left(-\lambda \tau_{u}\right)\right)=\exp \left(-u 2^{\alpha} \Gamma(\alpha+1) \int_{0}^{\infty} \frac{d v}{v^{\alpha+1}}\left(1-e^{-\lambda v}\right) e^{-\beta v}\right)
$$

and elementary computations yield:

$$
\begin{aligned}
E_{0}^{(-\alpha), \beta \downarrow}\left(\exp \left(-\lambda \tau_{u}\right)\right) & =\exp \left(-u 2^{\alpha} \Gamma(\alpha+1) \frac{\Gamma(\alpha-1)}{\alpha}\left\{(\lambda+\beta)^{\alpha}-\beta^{\alpha}\right\}\right) \\
& =\exp \left(-u 2^{\alpha} \frac{\pi}{\sin (\pi \alpha)}\left\{(\lambda+\beta)^{\alpha}-\beta^{\alpha}\right\}\right)
\end{aligned}
$$

As a consequence of these computations, it follows that, on one hand:

$$
E_{0}^{(-\alpha)}\left(\exp \left(-\lambda \tau_{u}\right)\right)=\exp \left(-u 2^{\alpha} \frac{\pi}{\sin (\pi \alpha)} \lambda^{\alpha}\right)
$$

\footnotetext{
${ }^{1}$ We shall also use the well-known general fact that such an absolute continuity relationship extends with $t$ replaced by any stopping time $T$ on the set $(T<\infty)$.
} 
and from (2.1) taken at $x=0$ and $t=\tau_{u}$, we can determine the constant $C_{\alpha}$ :

$$
C_{\alpha}=\frac{\pi}{\sin (\pi \alpha)} \text {. }
$$

On the other hand,

$$
\begin{aligned}
\lim _{\alpha \longrightarrow 0} E_{0}^{(-\alpha), \beta \downarrow}\left(\exp \left(-\lambda \tau_{u}\right)\right) & =\exp (-u\{\ln (\lambda+\beta)-\ln (\beta)\}) \\
& =\frac{1}{(1+\lambda / \beta)^{u}}
\end{aligned}
$$

Thus, assuming that we may represent the LHS of (2.3) as $E_{0}^{0, \beta \downarrow}\left(e^{-\lambda \tau_{u}}\right)$ for the law $P_{0}^{0, \beta \downarrow}$ of some diffusion $\operatorname{BES}(0, \beta \downarrow)$, instantaneously reflecting at 0 , we will thus have obtained a Krein representation of the Gamma process with parameter $\beta$, i.e. the subordinator whose Laplace transform in $\lambda$, at time $u$, is given by $(2.3)$.

Theorem 2.1. 1) For every $\beta>0$, there exists a diffusion $B E S(0, \beta \downarrow)$ on $\mathbb{R}_{+}$, which is instantaneously reflecting at 0 , and whose infinitesimal generator on $(0, \infty)$ is given by:

$$
\frac{1}{2} \frac{d^{2}}{d x^{2}}+\left(\frac{1}{2 x}+\sqrt{2 \beta} \frac{K_{0}^{\prime}}{K_{0}}(\sqrt{2 \beta} x)\right) \frac{d}{d x} .
$$

2) For some choice of the local time at 0 of $B E S(0, \beta \downarrow)$, the corresponding Itô measure of excursions may be described as:

a) $n_{0}^{\beta \downarrow}(V(e) \in d v)=\frac{1}{v} \exp (-\beta v) d v$

b) Conditionally on $V=v$, the process $(e(u), u \leq v)$ is distributed as a Bessel bridge of dimension 2 , and length $v$.

3) Let $x>0$, the following relation holds:

$$
\left.P_{x}^{(0), \beta \downarrow}\right|_{\mathcal{R}_{t} \cap\left(t<T_{0}\right)}=\left.\frac{K_{0}\left(\theta R_{t}\right)}{K_{0}(\theta x)} \exp (-\beta t) P_{x}^{(0)}\right|_{\mathcal{R}_{t}} .
$$

Note. The following relation, where we have extended $\left(1 / K_{0}\right)$ to $[0, \infty[$, with $\left(1 / K_{0}\right)(0)=0$, is equivalent to $(2.4)$ :

$$
\left.P_{x}^{(0)}\right|_{\mathcal{R}_{t}}=\left.\frac{K_{0}(\theta x)}{K_{0}\left(\theta R_{t \wedge T_{0}}\right)} \exp (\beta t) P_{x}^{(0), \beta \downarrow}\right|_{\mathcal{R}_{t}}
$$

The following theorem exhibits an absolute continuity relationship between the laws $P_{x}^{(0),(\beta+\gamma) \downarrow}$ and $P_{x}^{(0), \beta \downarrow}$ from which several important Laplace transforms may be immediately obtained. 
Theorem 2.2. Let $\beta, \gamma>0$, and $x>0$; then, there is the relationship:

$$
\begin{aligned}
& \left.P_{x}^{(0),(\beta+\gamma) \downarrow}\right|_{\mathcal{R}_{t}}= \\
& \left.\quad \frac{K_{0}\left(\sqrt{2(\beta+\gamma)} R_{t}\right)}{K_{0}\left(\sqrt{2 \beta} R_{t}\right)} \frac{K_{0}(\sqrt{2 \beta} x)}{K_{0}(\sqrt{2(\beta+\gamma)} x)}\left(1+\frac{\gamma}{\beta}\right)^{l_{t}} \exp (-\gamma t) P_{x}^{(0), \beta \downarrow}\right|_{\mathcal{R}_{t}} .
\end{aligned}
$$

In particular, for $x=0$ and $t$ being replaced by $\tau_{l}$, formula (2.6) simplifies as:

$$
\left.P_{0}^{(0),(\beta+\gamma) \downarrow}\right|_{\mathcal{R}_{t}}=\left.\frac{K_{0}\left(\sqrt{2(\beta+\gamma)} R_{t}\right)}{K_{0}\left(\sqrt{2 \beta} R_{t}\right)}\left(1+\frac{\gamma}{\beta}\right)^{l_{t}} \exp (-\gamma t) P_{0}^{(0), \beta \downarrow}\right|_{\mathcal{R}_{t}}
$$

and

$$
\left.P_{0}^{(0),(\beta+\gamma) \downarrow}\right|_{\mathcal{R}_{\tau_{l}}}=\left.\left(1+\frac{\gamma}{\beta}\right)^{l} \exp \left(-\gamma \tau_{l}\right) P_{x}^{(0), \beta \downarrow}\right|_{\mathcal{R}_{\tau_{l}}} .
$$

Note, also from (2.6), that the measure:

$$
\left.\frac{K_{0}(\sqrt{2 \beta} x)}{K_{0}\left(\sqrt{2 \beta} R_{t}\right)} \beta^{-l_{t}} \exp (\beta t) P_{x}^{(0), \beta \downarrow}\right|_{\mathcal{R}_{t}}
$$

does not depend on $\beta$.

Here are some consequences of $(2.6),(2.7),(2.8)$ :

- As a consequence of (2.6), one obtains the Laplace transform of $T_{0}=$ $\inf \left\{t ; R_{t}=0\right\}$ under $P_{x}^{(0), \beta \downarrow}$ :

$$
E_{x}^{(0), \beta \downarrow}\left(\exp \left(-\gamma T_{0}\right)\right)=\frac{K_{0}(\sqrt{2(\beta+\gamma)} x)}{K_{0}(\sqrt{2 \beta} x)} .
$$

In fact, $T_{0}$ is distributed ${ }^{2}$ as a $G I G(0 ; x, \sqrt{2 \beta})$ variable, i.e. with density:

$$
\frac{1}{2 K_{0}(x \sqrt{2 \beta})} \exp \left(-\frac{1}{2}\left(x^{2} / t+2 \beta t\right)\right) d t \text {. }
$$

As a consequence of (2.8), we obtain our main result:

Corollary 2.3. $\quad\left(\tau_{l} ; l \geq 0\right)$ is, under $P_{0}^{(0), \beta \downarrow}$, a Gamma process with parameter $\beta$, i.e. $\left(\beta \tau_{l}: l \geq 0\right)$ is a standard Gamma process:

$$
E_{0}^{(0), \beta \downarrow}\left(\exp \left(-\gamma \tau_{l}\right)\right)=\frac{1}{\left(1+\frac{\gamma}{\beta}\right)^{l}} \text { for } \gamma \geq 0 .
$$

\section{§3. Esscher and Girsanov Transforms}

This section is devoted to the proofs of the assertions contained in Section 2 .

\footnotetext{
${ }^{2}$ For many references to, and applications of, GIG variables, see Matsumoto-Yor [18].
} 
(3.1) To begin with, we explain how, once we have obtained a Krein representation for a subordinator $\left(S_{t}\right)$, we can obtain a related one for a second subordinator $\left(\tilde{S}_{t}\right)$ whose law is an Esscher transform of that of $\left(S_{t}\right)$. We consider a positive diffusion $\left(X_{t} ; t \geq 0\right)$ with 0 as an instantaneous reflecting boundary. We denote by $L_{t}$ a choice of its local time at 0 and $\left(\tau_{l} ; l \geq 0\right)$ the corresponding inverse local time. We are looking for a diffusion $\tilde{X}$ on $\mathbb{R}_{+}$with laws $\tilde{P}_{x}$ such that:

$$
\left.\tilde{P}_{0}\right|_{\mathcal{F}_{\tau_{u}}}=\left.\exp \left(\psi(a) u-a \tau_{u}\right) P_{0}\right|_{\mathcal{F}_{\tau_{u}}}
$$

for $a>0$, where $\psi$ denotes the Laplace exponent of the subordinator $\left(\tau_{u} ; u \geq 0\right)$. From (3.1), the inverse local time $\tilde{\tau}$ of $\tilde{X}$ is the Esscher transform $\tau^{(a)}$ of $\tau$ defined by:

$$
E_{0}\left[\exp \left(-\lambda \tau_{u}^{(a)}\right)\right]=\frac{E_{0}\left[\exp \left(-(\lambda+a) \tau_{u}\right)\right]}{E_{0}\left[\exp \left(-a \tau_{u}\right)\right]} .
$$

The Lévy measure $m^{(a)}(d x)$ of $\tau^{(a)}$ is related to the Lévy measure $m(d x)$ of $\tau$ by $m^{(a)}(d x)=\exp (-a x) m(d x)$ (see [7], [26], Chapter VII, Section 3c).

To define $\tilde{P}_{x}$, we need to compute the martingale

$$
\begin{gathered}
M_{s}=E\left[\exp \left(-a \tau_{t}\right) \mid \mathcal{F}_{s}\right], s \leq \tau_{t} \\
M_{s}=\exp (-a s) E_{X_{s}(\omega)}\left[\exp \left(-a \tau_{t-L_{s}(\omega)}\right)\right]
\end{gathered}
$$

Now, we have:

$$
E_{x}\left[\exp \left(-a \tau_{v}\right)\right]=E_{x}\left[\exp \left(-a T_{0}\right)\right] E_{0}\left[\exp \left(-a \tau_{v}\right)\right]
$$

and therefore:

$$
\frac{M_{s}}{M_{0}}=\varphi_{a \downarrow}\left(X_{s}\right) \exp \left(\psi(a) L_{s}-a s\right)
$$

where $\varphi_{a \downarrow}$ denotes the function defined by

$$
\varphi_{a \downarrow}(x)=E_{x}\left[\exp \left(-a T_{0}(X)\right)\right] \quad\left(\varphi_{a \downarrow}(0)=1\right)
$$

where $T_{0}(X)$ is the first hitting time of 0 by $X$. More generally, for $x \geq 0$, we define the law $\tilde{P}_{x}$ via the absolute continuity relation:

$$
\left.\tilde{P}_{x}\right|_{\mathcal{F}_{t}}=\left.\frac{\varphi_{a \downarrow}\left(X_{t}\right)}{\varphi_{a \downarrow}(x)} \exp \left(\psi(a) L_{t}-a t\right) P_{x}\right|_{\mathcal{F}_{t}}
$$

The generators $L$ and $\tilde{L}$ of $X$ and $\tilde{X}$ respectively are linked by:

$$
\tilde{L}=L+\frac{\varphi_{a \downarrow}^{\prime}(x)}{\varphi_{a \downarrow}(x)} \frac{d}{d x} .
$$




\section{(3.2) Examples:}

(1) $P_{x}=P_{x}^{(-\alpha)}, 0<\alpha<1$. Then,

$$
\varphi_{a \downarrow}(x)=c_{\alpha} \hat{K}_{\alpha}(\sqrt{2 a} x), \quad \psi(a)=\frac{\pi}{\sin (\pi \alpha)}(2 a)^{\alpha}
$$

and $\tilde{P}_{x}=P_{x}^{(-\alpha), a \downarrow}$.

(2) $P_{x}=P_{x}^{(-\alpha), \beta \downarrow}, 0<\alpha<1, \beta>0$. Then,

$$
\varphi_{a \downarrow}(x)=c_{\alpha}^{\prime} \frac{\hat{K}_{\alpha}(\sqrt{2(\beta+a)} x)}{\hat{K}_{\alpha}(\sqrt{2 \beta} x)} ; \quad \psi(a)=\frac{\pi}{\sin (\pi \alpha)}\left\{(2 a+2 \beta)^{\alpha}-(2 \beta)^{\alpha}\right\}
$$

and $\tilde{P}_{x}=P_{x}^{(-\alpha),(\beta+a) \downarrow}$.

From [3] and [20], we know that the inverse local time of $B E S(-\alpha)$ is a stable subordinator of index $\alpha$. It follows from subsection (3.1) and Example 1 above that the inverse local time of $B E S(-\alpha, \beta \downarrow)$ is a subordinator $S^{\alpha, \beta}$. The description of Itô's excursion measure of $B E S(-\alpha, \beta \downarrow)$ follows from the description of the excursion measure for $B E S(-\alpha)$ given in [3] and the Esscher transform. Note that we have not chosen the same normalisation for the local time as in [3]; we have the following relation between the two local time processes (from (2.2) and subsection 3.2 in [3]):

$$
l_{t}^{B Y}=\frac{\left(2^{\alpha} \Gamma(\alpha)\right)^{2}}{2(1-\alpha)} l_{t}^{D Y}
$$

where $l_{t}^{B Y}$ denotes the local time considered in [3], and $l_{t}^{D Y}$ denotes our present choice. Note that there is a mistake in [3] after (3.i) due to the identification of $\tau_{t}^{(-1 / 2)}$ (the inverse local time of the reflected Brownian motion) with $\tau_{t}$ (for the Brownian motion) instead of $\tau_{t / 2}$ and the correct formula (see p.45, after $(3 . i))$ is

$$
E\left[\exp \left(-k \tau_{t c(1 / 2,2 \nu)}^{(-\nu)}\right)\right]=\exp \left(-t c_{\nu} 2^{\nu-1} k^{\nu}\right)
$$

\section{(3.3) Proof of Theorems 2.1 and 2.2}

1) We consider the diffusion $X$ on $[0, \infty[$ with generator

$$
\frac{1}{2} \frac{d^{2}}{d x^{2}}+\left(\frac{1}{2 x}+\sqrt{2 \beta} \frac{K_{0}^{\prime}}{K_{0}}(\sqrt{2 \beta} x)\right) \frac{d}{d x} .
$$

A pair $(s(x), m(d x))$ of scale function and speed measure is given by:

$$
s(x)=\int_{0}^{x} \frac{d y}{y K_{0}^{2}(\sqrt{2 \beta} y)}, m(d x)=2 x K_{0}^{2}(\sqrt{2 \beta} x) d x .
$$


Note that $K_{0}(y) \sim_{0} \ln (2 / y)$, thus $\frac{1}{y K_{0}^{2}(\sqrt{2 \beta} y)}$ is integrable in 0 and $s(0)=0$. In order to obtain the behavior of $X$ at the boundary point 0 , we shall apply the criterium of Rogers-Williams [25, V.51] to the diffusion in natural scale $Y=s(X)$. The speed measure of $Y$ is given by:

$$
m_{Y}(d x)=\frac{2}{\left(s^{\prime} \circ s^{-1}(x)\right)^{2}} d x .
$$

Then, $\int_{0+} x m_{Y}(d x)<\infty$ implying that $T_{0}(Y)<\infty P_{x}$ p.s. for all $x>0$, and $\int_{0+} m(d x)<\infty$ ensuring that 0 is reflecting.

The description of the Itô measure follows from that of $B E S(-\alpha, \beta \downarrow)$ letting $\alpha \longrightarrow 0$.

The absolute continuity relation (2.4) is given in Pitman-Yor [21]. We can also obtain it from (2.1) and:

$$
\left.P_{x}^{(+\alpha)}\right|_{\mathcal{R}_{t}}=\left.\left(\frac{R_{t \wedge T_{0}}}{x}\right)^{2 \alpha} P_{x}^{(-\alpha)}\right|_{\mathcal{R}_{t}}, x>0 .
$$

Thus,

$$
\left.\left(\frac{R_{t \wedge T_{0}}}{x}\right)^{2 \alpha} P_{x}^{(-\alpha), \beta \downarrow}\right|_{\mathcal{R}_{t}}=\left.\frac{\hat{K}_{\alpha}\left(\theta R_{t}\right)}{\hat{K}_{\alpha}(\theta x)} \exp (-\beta t) P_{x}^{(+\alpha)}\right|_{\mathcal{R}_{t}}
$$

and letting $\alpha \longrightarrow 0$,

$$
\left.1_{\left(t<T_{0}\right)} \cdot P_{x}^{(0), \beta \downarrow}\right|_{\mathcal{R}_{t}}=\left.\frac{K_{0}\left(\theta R_{t}\right)}{K_{0}(\theta x)} \exp (-\beta t) P_{x}^{(0)}\right|_{\mathcal{R}_{t}} .
$$

The absolute continuity relation (2.6) follows from the example (3.2) 2 . with $\alpha=0$. In this case,

$$
\varphi_{a \downarrow}(x)=C \frac{K_{0}(\sqrt{2(\beta+a)} x)}{K_{0}(\sqrt{2 \beta} x)} ; \quad \psi(a)=\ln \left(1+\frac{a}{\beta}\right) .
$$

The other formulas follow easily.

\section{$\S 4$. Brownian Representations of the Subordinators $S^{\alpha, \beta}$}

We keep the same normalisation as in Section 2, i.e. the Laplace transform of a stable process is given by (2.2). 


\section{Proposition 4.1.}

(1) The stable subordinator $S^{\alpha}$ can be represented by the Brownian additive functional (see [3])

$$
S_{t}^{\alpha}=A_{\alpha}\left(\tau_{\frac{\Gamma(\alpha)^{2} t}{\alpha^{2 \alpha-1}}}\right)
$$

where

$$
A_{\alpha}(s):=\int_{0}^{s}\left|B_{u}\right|^{\frac{1}{\alpha}-2} d u, s \geq 0,
$$

and $\tau_{t}$ is the inverse local time of $B$.

(2) The subordinator $S^{\alpha, 1}$ can be represented as:

$$
\int_{0}^{\tau_{t}} h_{\alpha}\left(\left|B_{r}\right|\right) d r
$$

where $h_{\alpha}(x)=2\left(s_{\alpha}^{-1}(2 x)\right)^{2} K_{\alpha}^{4}\left(s_{\alpha}^{-1}(2 x)\right)$ and

$$
s_{\alpha}(x)=\int_{0}^{x} \frac{d y}{y K_{\alpha}^{2}(y)}
$$

(3) The Gamma process $S^{0,1}$ has the following representation:

$$
\left(\int_{0}^{\tau_{t}} h\left(\left|\beta_{u}\right|\right) d u ; t \geq 0\right)
$$

with

$$
h(x)=2\left(G^{-1}(2 x)\right)^{2} K_{0}^{4}\left(G^{-1}(2 x)\right),
$$

where $G^{-1}$ is the inverse of the increasing function $G$ given by:

$$
G(z)=\int_{0}^{z} \frac{d x}{x K_{0}^{2}(x)}
$$

\section{Sketch of Proof:}

1) The first point has been obtained in [3]. More precisely,

$$
E\left[\exp \left(-\frac{\lambda}{2} A_{\alpha}\left(\tau_{t}\right)\right)\right]=\exp \left(-t c_{\alpha} \lambda^{\alpha}\right), \quad \lambda \geq 0,
$$

where

$$
c_{\alpha}=\frac{\pi}{\alpha \sin (\pi \alpha)}\left(\frac{\alpha^{\alpha}}{\Gamma(\alpha)}\right)^{2} .
$$


2) By a Girsanov transform, we can find a diffusion $X$ such that the subordinator

$$
A_{\alpha}^{X}\left(\tau_{t}\right):=\int_{0}^{\tau_{t}(X)}\left|X_{u}\right|^{\frac{1}{\alpha}-2} d u
$$

is a Esscher transform of the stable subordinator $A_{\alpha}\left(\tau_{t}\right)$ and thus is distributed as $S^{\alpha, 1}$ (up to a constant). Then, we write the diffusion in natural scale $s_{\alpha}(X)$ as a time-change of Brownian motion. We can also start from $B E S(\alpha, 1 \downarrow)$ and use a time change method.

3) By an application of Itô's formula, we can prove that:

$$
G^{-1}\left(2\left|B_{t}\right|\right)=Y\left(\int_{0}^{t} 2 h\left(\left|B_{s}\right|\right) d s\right)
$$

where $Y$ is a $B E S(0,1 \downarrow)$ process from which we can deduce that $\tau_{t}(Y) \stackrel{(\text { law })}{=}$ $\int_{0}^{\tau_{t}(B)} 2 h\left(\left|B_{s}\right|\right) d s$ where $\tau_{t}(Y)$ is the inverse diffusion local time of $Y$, defined with the speed measure $m_{Y}(d y)=4 y K_{0}^{2}(y) d y$.

\section{$\S 5 . \quad$ Some Complements}

(5.1) The case $\alpha=1 / 2$. Let us go back to the representation (4.2) for the subordinator with Lévy measure $k_{\alpha}^{\prime} \exp \left(-\frac{y}{2}\right) \frac{d y}{y^{1+\alpha}}$. In the case $\alpha=1 / 2,(4.2)$ becomes:

$$
\int_{0}^{\tau_{t}} \frac{d r}{\left(1+2\left|\beta_{r}\right|\right)^{2}}
$$

We now explain how this representation may be reduced to the more classical one, as given in (5.2) below. For this purpose, we recall that the stable subordinator of index $1 / 2$ can also be realized as the distribution of $\left(T_{t} ; t \geq 0\right)$ where $T_{t}$ denotes the first hitting time of $t$ by a Brownian motion, starting from 0 . We have the same interpretation for its Esscher transform. Indeed,

$$
\begin{aligned}
\ln \left(1+2\left|B_{t}\right|\right) & =2\left[\int_{0}^{t} \frac{\operatorname{sgn}\left(B_{s}\right) d B_{s}}{\left(1+2\left|B_{s}\right|\right)}-\int_{0}^{t} \frac{d s}{\left(1+2\left|B_{s}\right|\right)^{2}}+L_{t}\right] \\
& =2\left[-\beta^{(1)}\left(\int_{0}^{t} \frac{d s}{\left(1+2\left|B_{s}\right|\right)^{2}}\right)+L_{t}\right]
\end{aligned}
$$

where $\left(\beta^{(1)}(u) ; u \geq 0\right)$ is a Brownian motion with drift 1 .

From Skorokhod's lemma, we derive:

$$
L_{t}=\sup \left\{\beta_{s}^{(1)} ; s \leq \int_{0}^{t} \frac{d r}{\left(1+2\left|B_{r}\right|\right)^{2}}\right\}
$$


Therefore, we obtain:

$$
\int_{0}^{\tau_{l}} \frac{d r}{\left(1+2\left|B_{r}\right|\right)^{2}}=\inf \left\{u ; \beta_{u}^{(1)}=l\right\}:=T_{l}^{(1)} .
$$

Thus, from (5.1), $\left(T_{l}^{(1)}, l \geq 0\right)$ is a subordinator with Lévy measure $C \exp \left(-\frac{y}{2}\right) \frac{d y}{y^{3 / 2}}$ and Laplace transform

$$
E\left(\exp \left(-\frac{\lambda^{2}}{2} T_{l}^{(1)}\right)\right)=\exp \left(-l\left(\sqrt{\lambda^{2}+1}-1\right)\right)
$$

Of course, the above Laplace transform could also have been computed from the Laplace transform of $T_{l}$ and the Cameron-Martin formula.

(5.2) Symmetric Lévy processes. From the Brownian representation of the Gamma process, we can give a Brownian representation for the symmetric Gamma process (or variance Gamma process) distributed as $\left(\gamma_{1}(t)-\gamma_{2}(t) ; t \geq\right.$ $0)$ where $\gamma_{1}$ and $\gamma_{2}$ are two independent Gamma processes. More generally, we have:

Proposition 5.1. Let $S_{1}$ and $S_{2}$ be two independent subordinators with Brownian representations:

$$
\left(S_{i}(t) ; t \geq 0\right) \stackrel{(\text { law })}{=}\left(\int_{0}^{\tau_{t}} d s \varphi_{i}\left(\left|B_{s}\right|\right) ; t \geq 0\right), i=1,2 .
$$

Then, we have the Brownian representation for $\left(S_{1}(t)-S_{2}(t) ; t \geq 0\right)$ as follows:

$$
\left(S_{1}(t)-S_{2}(t) ; t \geq 0\right) \stackrel{(\text { law })}{=}\left(\int_{0}^{\tau_{2 t}} d s \varphi\left(B_{s}\right) ; t \geq 0\right)
$$

where $\varphi(x)=\left\{\begin{array}{l}\varphi_{1}(x), x>0 \\ -\varphi_{2}(-x), x<0\end{array}\right.$.

Proof. It suffices to write

$$
\int_{0}^{\tau_{t}} d s \varphi\left(B_{s}\right)=\int_{0}^{\tau_{t}} d s 1_{\left(B_{s}>0\right)} \varphi_{1}\left(B_{s}\right)-\int_{0}^{\tau_{t}} d s 1_{\left(B_{s}<0\right)} \varphi_{2}\left(-B_{s}\right) .
$$

Then, we use the representation

$$
B_{s}^{+}=\left|\beta^{(+)}\right| \int_{0}^{s} 1_{\left(B_{u}>0\right)} d u, B_{s}^{-}=\left|\beta^{(-)}\right| \int_{0}^{s} 1_{\left(B_{u}<0\right)} d u
$$


where $\beta^{(+)}$and $\beta^{(-)}$are (as a consequence of Knight's theorem) two independent Brownian motions ${ }^{3}$; see, e.g., proofs of the arc sine law for Brownian motion, inspired from D. Williams [34] (see [9], [19]).

Then, we can write

$$
\int_{0}^{\tau_{t}} d s \varphi\left(B_{s}\right)=\int_{0}^{A_{\tau_{t}}^{(+)}} d h \varphi_{1}\left(\left|\beta_{h}^{(+)}\right|\right)-\int_{0}^{A_{\tau_{t}}^{(-)}} d h \varphi_{2}\left(\left|\beta_{h}^{(-)}\right|\right)
$$

where

$$
A_{\tau_{t}}^{(+)}=\int_{0}^{\tau_{t}} d s 1_{\left(B_{s}>0\right)} d s ; \quad A_{\tau_{t}}^{(-)}=\int_{0}^{\tau_{t}} d s 1_{\left(B_{s}<0\right)} d s,
$$

and moreover, from (5.4), we also learn that:

$$
A_{\tau_{t}}^{(+)}=\tau_{t / 2}\left(\beta^{(+)}\right), A_{\tau_{t}}^{(-)}=\tau_{t / 2}\left(\beta^{(-)}\right) .
$$

Finally, we have obtained the result.

(5.3) The Itô measure of $B E S(-\alpha, \beta \downarrow)$. The description of the Itô measure given in Section 2 relies upon one of the descriptions of the Itô measure $n_{\alpha}$ of $B E S(-\alpha)$ given in [3]. There is a second description of $n$ conditionally to the maximum of the excursion.

Proposition 5.2. a) Under $n_{\alpha}^{\beta \downarrow}$, the distribution of $M$ satisfies:

$$
n_{\alpha}^{\beta \downarrow}(M \geq x)=2(2 \beta)^{\alpha} \frac{K_{\alpha}(\sqrt{2 \beta} x)}{I_{\alpha}(\sqrt{2 \beta} x)} .
$$

b) Conditionally on $M=x$, the maximum is attained at a unique time $R$ and the processes $\left(e_{t} ; 0 \leq t \leq R\right)$ and $\left(e_{V-t}, 0 \leq t \leq V-R\right)$ are two independent $B E S(\alpha, \beta \uparrow)$ processes, starting from 0 , stopped at their first hitting time $T_{x}$ of $x$.

Proof. a) From the description of $n_{\alpha}^{\beta \downarrow}$ given in section 2, we have:

$$
n_{\alpha}^{\beta \downarrow}\left[f\left(M^{2}\right)\right]=2^{\alpha} \Gamma(\alpha+1) \int_{0}^{\infty} \frac{\exp (-\beta v)}{v^{\alpha+1}} \Pi_{v}^{(\alpha)}\left[f\left(M^{2}\right)\right] d v
$$

where $\Pi_{v}^{(\alpha)}$ is the distribution of a Bessel bridge of index $\alpha$ and length $v$.

$$
n_{\alpha}^{\beta \downarrow}\left[f\left(M^{2}\right)\right]=2^{\alpha} \Gamma(\alpha+1) \int_{0}^{\infty} \frac{\exp (-\beta v)}{v^{\alpha+1}} \Pi_{1}^{(\alpha)}\left[f\left(v M^{2}\right)\right] d v .
$$

\footnotetext{
${ }^{3}$ However, we emphasize that, knowing $B$, only the reflected Brownian motions $\left|\beta_{h}^{(+)}\right|$and $\left|\beta_{h}^{(-)}\right|$are accessible, and not $\beta^{(+)}$and $\beta^{(-)}$.
} 
Now, from [22, Theorem 3.1]

$$
\Pi_{1}^{(\alpha)}[\phi(r)]=c_{\alpha} E\left[\phi(\tilde{R})(\tilde{M})^{-2 \alpha}\right]
$$

where the process $\tilde{R}$ is defined by

$$
\tilde{R}_{t}=(T+\hat{T})^{-1 / 2} Y_{t(T+\hat{T})}, 0 \leq t \leq 1
$$

and $Y$ is the process connecting the paths of two independent $B E S(2+2 \alpha)$ processes $R$ on $[0, T]$ (first hitting time of 1 ) and $\hat{R}$ on $[0, \hat{T}]$ back to back, i.e.

$$
Y_{t}= \begin{cases}R_{t} & t \leq T \\ \hat{R}_{T+\hat{T}-t} & T \leq t \leq T+\hat{T}\end{cases}
$$

Then,

$$
\tilde{M}:=\sup _{s \leq 1} \tilde{R}_{s}=(T+\hat{T})^{-1 / 2}
$$

and $c_{\alpha}=2^{\alpha} \Gamma(\alpha+1)$.

It follows that:

$$
\Pi_{1}^{(\alpha)}\left[f\left(v M^{2}\right)\right]=c_{\alpha} E\left[f\left(\frac{v}{T+\hat{T}}\right)(T+\hat{T})^{\alpha}\right] .
$$

Thus,

$$
\begin{aligned}
n_{\alpha}^{\beta \downarrow}\left[f\left(M^{2}\right)\right] & =2^{\alpha} \Gamma(\alpha+1) c_{\alpha} E\left[\int_{0}^{\infty} \frac{\exp (-\beta w(T+\hat{T}))}{w^{\alpha+1}} f(w) d w\right] \\
& =2^{\alpha} \Gamma(\alpha+1) c_{\alpha} \int_{0}^{\infty} \frac{f(w)}{w^{\alpha+1}} E[\exp (-\beta w(T+\hat{T}))] d w \\
& =\left[2^{\alpha} \Gamma(\alpha+1)\right]^{-1} c_{\alpha} \int_{0}^{\infty} \frac{f(w)}{w^{\alpha+1}}\left(\frac{(\sqrt{2 \beta w})^{\alpha}}{I_{\alpha}(\sqrt{2 \beta w})}\right)^{2} d w \\
& =(2 \beta)^{\alpha} \int_{0}^{\infty} \frac{f(w)}{w I_{\alpha}^{2}(\sqrt{2 \beta w})} d w \\
& =2(2 \beta)^{\alpha} \int_{0}^{\infty} \frac{f\left(y^{2}\right)}{y I_{\alpha}^{2}(\sqrt{2 \beta} y)} d y
\end{aligned}
$$

Therefore, we obtain

$$
n_{\alpha}^{\beta \downarrow}(M \in d y)=2(2 \beta)^{\alpha} \frac{1}{y I_{\alpha}^{2}(\sqrt{2 \beta} y)} d y
$$

hence:

$$
\begin{aligned}
n_{\alpha}^{\beta \downarrow}(M \geq x) & =2(2 \beta)^{\alpha} \int_{x}^{\infty} \frac{1}{y I_{\alpha}^{2}(\sqrt{2 \beta} y)} d y \\
& =2(2 \beta)^{\alpha} \frac{K_{\alpha}(\sqrt{2 \beta} x)}{I_{\alpha}(\sqrt{2 \beta} x)} .
\end{aligned}
$$


We also refer to [24] for related computations.

As a verification, we can let $\beta \longrightarrow 0$ to obtain:

$$
n_{\alpha}(M \geq x)=2^{2 \alpha} \Gamma(\alpha) \Gamma(\alpha+1) \frac{1}{x^{2 \alpha}} .
$$

This agrees with the description of the distribution of $M$ under $\hat{n}_{\alpha}$ given in Biane-Yor [3]:

$$
\hat{n}_{\alpha}(M \geq x)=\frac{1}{x^{2 \alpha}}
$$

and from our normalisation given in Section 2,

$$
n_{\alpha}=2^{2 \alpha} \Gamma(\alpha) \Gamma(\alpha+1) \hat{n}_{\alpha} .
$$

b) We refer to [21] for the definition of Bessel processes with drift $B E S(\alpha, \beta \uparrow)$. The description of $n_{\alpha}^{\beta \downarrow}$, conditionally to $M$, follows from the description of $n_{\alpha}$ (see [3]), the relation $n_{\alpha}^{\beta \downarrow}(d e)=\exp (-\beta V(e)) n_{\alpha}(d e)$ and

$$
\left.P_{0}^{(\alpha), \beta \uparrow}\right|_{\mathcal{F}_{T_{x}}}=\left.C_{\alpha} x^{-\alpha} I_{\alpha}(\theta x) \exp \left(-\beta T_{x}\right) P_{0}^{(\alpha)}\right|_{\mathcal{F}_{T_{x}}}
$$

(see Proposition 3.1 in [21]).

(5.4) A density of occupation formula for $B E S(-\alpha, \beta \downarrow)$. Although in the discussion made throughout this paper, the local times at levels $y \neq$ 0 with respect to the different diffusion processes of interest to us, did not intervene, it may be useful for some possible extensions of our study that we now present formulae of occupation densities which are in agreement with our previous choices of the local times at 0 . In the general study of one-dimensional diffusions, it is customary to choose the family of local times of, say, the diffusion $R$, such that they satisfy the density of occupation formula: for every $h$ : $\mathbb{R}_{+} \longrightarrow \mathbb{R}_{+}$, Borel,

$$
\int_{0}^{t} h\left(R_{s}\right) d s=\int_{0}^{\infty} h(y) L_{t}^{y} m(d y)
$$

(we assume $R$ here takes values in $\mathbb{R}_{+}$) where $m(d y)$ is a speed measure for $R$. There exists a continuous family in $(y, t)$ of such local times (see [8]). Precisely, for $R$ a $B E S(-\alpha, \beta \downarrow)$, we have chosen in (5.6):

$$
m_{\alpha}^{\beta \downarrow}(d y)=4 y^{1-2 \alpha} \hat{K}_{\alpha}^{2}(\sqrt{2 \beta} y) d y
$$

where $\hat{K}_{\alpha}(y)=y^{\alpha} K_{\alpha}(y)$, which satisfies $\hat{K}_{\alpha}(0)=2^{\alpha-1} \Gamma(\alpha)$ for $\alpha>0$, and formula (5.7) is valid also for $\beta=0$. For $\alpha=0$, we need to take $\beta>0$ and formula (5.7) becomes:

$$
m_{0}^{\beta \downarrow}(d y)=2 y K_{0}^{2}(\sqrt{2 \beta} y) d y .
$$


From (5.6), and the continuity of the local times, there is the formula:

$$
L_{t}^{0}=\lim _{\varepsilon \longrightarrow 0} \frac{1}{m([0, \varepsilon])} \int_{0}^{t} 1_{\left(R_{s} \leq \varepsilon\right)} d s .
$$

It follows from this approximation and the consequence of the formulae (5.7) and (5.8):

$$
\begin{array}{ll}
\text { - for } \alpha>0, & m_{\alpha}^{\beta \downarrow}([0, \varepsilon]) \\
\text { - for } \alpha=0, \quad m_{0}^{\beta \downarrow}([0, \varepsilon]) \underset{\varepsilon}{\sim} m_{\alpha}([0, \varepsilon]) & \\
\longrightarrow & m_{0}^{\gamma \downarrow}([0, \varepsilon]) \quad \text { for every } \beta, \gamma>0,
\end{array}
$$

that the local times at 0

- for $B E S(-\alpha, \beta \downarrow)$ and $B E S(-\alpha)$ on one hand

- for $B E S(0, \beta \downarrow)$ and $B E S(0, \gamma \downarrow)$ on the other hand

are defined in a coherent manner from (5.9) under these pairs of locally equivalent diffusion laws.

\section{References}

[1] Bertoin, J., Applications de la théorie spectrale des cordes vibrantes aux fonctionnelles additives principales d'un mouvement brownien réfléchi, Ann. Inst. H. Poincaré Probab. Statist., 25 (1989), 307-323.

[2] Subordinators: examples and applications, Lectures on Probability Theory and Statistics, Ecole d'Eté de Probabilités de Saint Flour XXVII-1997, Lecture Notes in Math., 1717, Springer, 1999.

[3] Biane, P. and Yor, M., Valeurs principales associées aux temps locaux browniens, Bull. Sci. Math., 111 (1987), 23-101.

[4] Donati-Martin, C. and Yor, M., On a Krein representation of the variance-gamma processes in terms of the $B E S(0, \beta \downarrow)$ processes. In preparation (2005).

[5] Donati-Martin, C., Roynette, B., Vallois, P. and Yor, M., On constants related to the choice of the local time at 0 and the corresponding Itô measure for Bessel processes with dimension $d=2(1-\alpha), 0<\alpha<1$, Prépublication de l'Institut E. Cartan, 2005. To appear in Studia Sci. Math. Hungar.

[6] Emery, M. and Yor, M., A parallel between Brownian bridges and gamma bridges, Publ. RIMS, Kyoto Univ., 40 (2004), 669-688.

[7] Esscher, F., On the probability function in the collective theory of risk, Scandinavisk Aktuarietidskrift, 15 (1932), 175-195.

[8] Ito, K. and McKean, H. P., Diffusion Processes and Their Sample Paths, Springer, Berlin, 1965.

[9] Karatzas, I. and Shreve, S. E., Brownian Motion and Stochastic Calculus, Second edition, Springer-Verlag, New York, 1991.

[10] Knight, F. B., Characterization of the Lévy measure of inverse local times of gap diffusion, Seminar on Stochastic Processes, 1981, 53-78, Prog. Prob. Statist., 1, Birkhäuser, 1981. 
[11] Kotani, S. and Watanabe, S., Krein's spectral theory of strings and generalized diffusion processes, Functional Analysis in Markov processes (Katata/Kyoto 1981), 235-259, LNM, 923, Springer, 1982.

[12] Küchler, U., On sojourn times, excursions and spectral measures connected with quasidiffusions, J. Math. Kyoto Univ., 26 (1986), 403-421.

[13] Küchler, U. and Salminen, P., On spectral measures of strings and excursions of quasi diffusions, Séminaire de Probabilités, XXIII, 490-502, Lecture Notes in Math., 1372, Springer, Berlin, 1989.

[14] Lebedev, N. N., Special Functions and Their Applications, Dover Publications, 1972.

[15] Madan, D. B., Carr, P. P. and Chang, E. C., The variance gamma process and option pricing, European Finance Review, 2 (1998), 79-105.

[16] Madan, D. B. and Milne, F., Option pricing with variance gamma martingale components. Math. Finance, 1 (1991), 39-56.

[17] Madan, D. B. and Seneta, E., The variance gamma model for share markets returns, $J$. Bus., 63 (1990), 511-524.

[18] Matsumoto, H. and Yor, M., An analogue of Pitman's $2 M-X$ theorem for exponential Wiener functionals. II. The role of the generalized inverse Gaussian laws, Nagoya Math. J., 162 (2001), 65-86.

[19] McKean, H. P., Brownian local times. Adv. Math., 16 (1975), 91-111.

[20] Molchanov, S. A. and Ostrovski, E., Symmetric stable processes as traces of degenerate diffusion processes, Theory Probab. Appl., 14 (1969), 128-131.

[21] Pitman, J. and Yor, M., Bessel processes and infinitely divisible laws, Stochastic integrals, Lecture Notes in Math., 851, Springer, Berlin, 1981.

[22] Decomposition at the maximum for excursions and bridges of one-dimensional diffusions, Itô's Stochastic calculus and Probability theory (Ikeda, N., Watanabe, S., Fukushima, M. and Kunita, H., eds.), Springer, 1996.

[23] dinator, Ann. Probab., 25 (1997), 855-900.

[24] Probab., 29 (2001), 361-384.

[25] Rogers, L. C. G and Williams, D., Diffusions, Markov Processes and Martingales, vol 2: Itô calculus, Wiley and Sons, New York, 1987.

[26] Shiryaev, A. N., Essentials of Stochastic Finance, World Scientific, Singapore, 1999.

[27] Vershik, A. and Yor, M., Multiplicativité du processus gamma et étude asymptotique des lois stables d'indice $\alpha$, lorsque $\alpha$ tend vers 0, Prépublication 289, Laboratoire de Probabilités, Paris 6, 1995.

[28] Tsilevich, N. and Vershik, A., Quasi-invariance of the gamma process and multiplicative properties of the Poisson-Dirichlet measures, C. R. Acad. Sci. Paris Sér. I Math., 329 (1999), 163-168.

[29] Tsilevich, N., Vershik, A. and Yor, M., An infinite-dimensional analogue of the Lebesgue measure and distinguished properties of the gamma process, J. Funct. Anal., 185 (2001), $274-296$.

[30] The Markov-Krein identity and the quasi-invariance of the gamma process, (Russian) Zap. Nauchn. Sem. S.-Peterburg. Otdel. Mat. Inst. Steklov. (POMI) 283 (2001), Teor. Predst. Din. Sist. Komb. i Algoritm. Metody. 6, 21-36, 258; translation in J. Math. Sci. (N. Y.), 121 (2004), 2303-2310.

[31] Watanabe, S., On time inversion of one-dimensional diffusion processes. Z. Wahrscheinlichkeitstheorie und Verw. Gebiete, 31 (1974/75), 115-124.

[32] , Invariants of one-dimensional diffusion processes and applications. International Conference on Probability Theory and its Applications (Taejon, 1998), J. Korean Math. Soc., 35 (1998), 637-658.

[33] Bilateral Bessel diffusion processes with drift and time inversion, preprint.

[34] Williams, D., Markov properties of Brownian local time, Bull. Amer. Math. Soc., 75 (1969), 1035-1036. 